\title{
Gerichtsurteil
}

\section{Direkte Empfehlung einer Krankenkasse durch den Hausarzt unzulässig}

Dass ein Hausarzt seinen Patienten einen Rat bezüglich der Wahl einer Krankenkasse gibt, gehört sicherlich zum Alltag. Gerade jetzt, wo eine Reihe von Krankenkassen einen Zusatzbeitrag erhebt, ist das Thema Kassenwechsel aktuell. Doch mit seinem Rat kann sich der Hausarzt leicht Ärger ins Haus holen.

— Enger werdender Honorarspielraum, geringer werdende Regelleistungsvolumina (RLV) auf der einen Seite und lukrative Hausarztverträge auf der anderen Seite bringen den Hausarzt schnell in eine Konfliktsituation. Insbesondere dann, wenn es um so verlockende Verträge geht wie in Bayern der Vertrag zwischen der AOK und dem Bayerischen Hauärzteverband.

Das Landgericht München gab dem Verband gegen unlauteren Wettbewerb Recht: Dieser hatte eine einstweilige Verfügung gegen den Hausärzteverband beantragt und im Hauptverfahren bestätigt bekommen.

Der Verband hatte seinen Hausärzten Infomaterial für die Patienten zur Verfügung gestellt. Darin wurde den Patienten ein Wechsel zum Hausarztvertrag der AOK Bayern schmackhaft gemacht. In der Einleitung hatte es zwar geheißen: „Wir bitten um Verständnis, dass wir Ihnen keine konkreten Empfehlungen geben dürfen." Aber dann war der Tenor doch richtungweisend: „Wir können Sie aber darüber informieren, dass die Bereitschaft der Kassen zur Sicherung Ihrer hausärztlichen Versorgung durch den Abschluss eines Hausarztvertrages nicht gleich hoch ist." Die positive Herausstellung des wegweisenden AOKVertrages war anderen Krankenkassen ein Dorn im Auge. Sie alarmierten die
Wettbewerbsschützer, die vor Gericht zogen, weil „... das Vertrauen des Patienten in seinen Arzt, den Hausarzt missbraucht werde“.

\section{Die Bedenken des Landgerichts}

Das Landgericht formulierte Bedenken: „Der bei einer anderen Kasse Versicherte, der im Wartezimmer gelesen hat, dass er zur AOK wechseln soll, um unter anderem die Existenz der haus-

\section{Studienzulassung}

Bundesgesundheitsminister Philipp Rösler (FDP) plant offenbar eine „Landarztquote“, um Engpässen in der ambulanten ärztlichen Versorgung in dünn besiedelten Regionen vorzubeugen.

Nach den Plänen Röslers sollen Medizinstudenten, die sich bereit erklären, nach Abschluss ihres Studiums eine Zeit lang als Landarzt zu arbeiten, bevorzugt einen Studienplatz erhalten.

Die „Landarztquote“ ist Teil eines umfangreichen Maßnahmenpakets, mit dem die Koalition dem drohenden Mangel an Ärzten im niedergelassenen, aber auch im Klinikbereich entgegenwirken will. Eckpunkte für einen entsprechenden Gesetzentwurf sollen noch vor der ärztlichen Versorgung sicherzustellen, muss befürchten, dass der Arzt ... ihm nicht mit der selben Haltung gegenübertritt wie einem AOK-Patienten."

Ärzte dürfen zwar über gesetzliche und vertragliche Rahmenbedingungen der gesetzlichen Krankenkassen informieren, dürfen aber nicht ausgesprochen forciert die Krankenkassenwahl steuern. Diese Anforderungen sind insbesondere bei schriftlichen Informationen penibel zu beachten, denn darüber, was einem Richter schwarz auf weiß vorliegt, über diesen Inhalt entscheidet dann nur noch der Richter.

H. WALBERT =

\section{Kommt eine Landarztquote?}

Sommerpause vorliegen. Rösler hatte sich zuvor auch dafür ausgesprochen, den Zugang zum Medizinstudium zu erleichtern und den geltenden Numerus clausus abzuschaffen.

Beim Koalitionspartner stießen die Pläne des FDP-Ministers auf Zustimmung. „Wir müssen kreativ Anreize setzen, dass Medizinstudenten sich für eine Weiterbildung zum Hausarzt entscheiden und anschließend auch auf das Land gehen mögen", sagte Unions-Gesundheitsexperte Jens Spahn. Dazu könne auch eine Bevorzugung von solchen Studenten gehören, „die neben anderen Qualifikationen sich für einige Jahre als Landarzt verpflichten“. ном . 